\title{
Preference gives more consistent judgments than oddity only if the task can be modeled as forced choice
}

\author{
E. N. GEELHOED and A. W. MACRAE \\ University of Birmingham, Birmingham, United Kingdom \\ and \\ DANIEL M. ENNIS \\ Philip Morris Research Center, Richmond, Virginia
}

\begin{abstract}
Earlier data showed that subjects presented with two samples of distilled water and one of tap water were significantly more consistent in choosing the tap water as preferred than in identifying it as the odd sample in the set. The results were sometimes interpreted as demonstrating greater sensitivity for hedonic judgments than for oddity judgments. They are now shown to be explained by the statistical properties of the decision rules followed in different judgment tasks. In a new experiment, oddity and preference judgments were obtained in a replication of the original task with extra conditions. In two of the new conditions, the decision structure of a preference task was the same as that for the oddity task; in these conditions, performance was no better than with explicit oddity responses. The Thurstone-Ura model of triangle judgments proposed by Frijters predicts the results as an outcome of the greater statistical power of three-alternative forced choice tests compared with triangular tests. An excellent fit to all the data is given by a model wherein all subjects have the same $d^{\prime}$ for the difference between the water types but $25 \%$ of them prefer distilled water to tap water.
\end{abstract}

In the triangle test, a subject is presented with three samples, two that are identical and one that is different; the task is to locate the different sample. It differs in important ways from three-alternative forced choice (3-AFC), and its principal virtue is that it does not require the subject to identify the attribute responsible for the difference between the samples. Thus, it is particularly suitable for complex stimuli, such as foods, and is often used in testing consumer products.

MacRae and Geelhoed (1992) reported an experiment in which subjects were given one sample of tap water and two samples of distilled water with instructions either to locate the odd sample or to state a preference for one of the samples. Just over half of the subjects selected tap water as the odd one, whereas almost three quarters (a significantly larger proportion) expressed a preference for tap water. To express consistent preferences, they must have been able to detect some difference between tap and distilled water. This result was surprising because any real

This work was supported by a research contract from the Ministry of Agriculture, Fisheries and Food. The results of the research are the property of the Ministry and are Crown copyright. E. N. Geelhoed is now with Hewlett Packard Laboratories, Bristol. Correspondence should be addresed to A. W. MacRae, School of Psychology, University of Birmingham, Edgbaston, Birmingham B15 2TT, England. differences that make preferences more pronounced should also aid identification of oddity. It is, however, perfectly legitimate for subjects to differ in their preferences, so preferences might be expected to be less consistent than oddity detections.

Several possible explanations were suggested for the effect, by ourselves and by others, and most of them related to the nature of hedonic judgments as compared with judgments of other differences. For example, a preference is neither right nor wrong, and because errors are therefore impossible, subjects may relax and discriminate better. Another possibility is that preferences are biologically more important than differences, with substantial neural capacity being dedicated to the selection of rewarding stimuli, whereas detection of mere difference is an incidental matter and dedicated processing is unlikely.

Another type of explanation focuses on the different decision rules of the tasks. Selecting the odd member from three may require comparison of three distances, whereas choosing the preferred one from three requires comparison of three magnitudes. According to a Thurstone model, distances have more variability than the individual magnitudes that define them and so are likely to be more often confused. An experiment was conducted to distinguish between the possibility of preference being intrinsically easier than oddity judgments and the possibility that the effect occurs because of different decision rules. 


\section{METHOD}

\section{Subjects}

The subjects were 96 volunteers, mostly recruited from visitors to our laboratory on two consecutive "open days." A few colleagues and students were added later to complete the balanced design.

\section{Materials}

Tap water was obtained from the same tap throughout the experiment. Distilled water was prepared in an automatic still. Before each testing day, fresh samples of each water were obtained and stored overnight in screw-topped 2-1 glass bottles to stabilize them and equalize their temperatures.

White expanded polystyrene thermal cups were used to present the samples. Each cup was labeled with a single green or blue spot marked on the molding boss on its base in such a way that even the presence of the spot was not visible until the cups were emptied and turned over for scoring. The water was served at a temperature of $15^{\circ} \pm 2^{\circ}$ Celsius equalized to better than $0.2^{\circ}$ within each set.

\section{Procedure}

The cups of water were dispensed from a screened mobile booth containing bar optics for metering the water. Each set of three cups was presented in a triangular group on a tray, with the odd cup positioned randomly. Instructions were printed on a plastic-coated card that covered the three samples when they were presented. They consisted of four numbered sentences of which the first three were the same on every card and a note, centered and in italics, at the bottom of the card that also was always the same.

The three unchanging sentences were:

1. The three samples are all water but they come from more than one source.

2. Taste the three samples, in any order and as often as you like.

3. They are perfectly safe to swallow but you may spit them out if you prefer.

Sentence 4 was different for each of four tasks, which we identify as A, B, C, and D, though these letters did not appear on the cards. They read:

A. When you have tasted them all, please pick out: one sample that seems most different from the other two.

B. When you have tasted them all, please pick out: one sample that you like most $O R$ one sample that you like least.

C. When you have tasted them all, please pick out: one sample that you like most.

D. When you have tasted them all, please pick out: one sample that you like least.

The note at the bottom of every card read:

(If in doubt, choose the one you are most sure about.)

The subjects were tested individually while seated at a table. A tray was placed in front of the subject, carrying four sets of three samples, each set covered with its instruction card. The subject removed the sets from the tray one at a time, working from the nearest to the farthest, read the instructions, tasted the samples, and selected one of them according to the instructions. The card was placed on top of the other two samples and the selected sample on top of the card. The structure so formed preserved the task instructions and the resulting judgment as a set until all four tasks were completed.

All 24 sequences of the four tasks were used four times. For half of the subjects, Tasks $A$ and $C$ were conducted with triangles of two tap and one distilled water, and Tasks B and D had one tap and two distilled. The others had the reverse arrangement. To min- imize recording errors, different colors of instruction cards and different colors of scoring forms were used for these two groups of subjects, who were tested approximately alternately.

\section{RESULTS}

Table 1 summarizes the results. From columns $\mathrm{C}$ and $D$, we see that tap water was generally preferred to distilled water, being selected as most preferred by $67(70 \%)$ of the subjects and as least preferred by $33(34 \%)$. Since every subject made a choice of most and least preferred sample from two separate sets of three, the same type of water might be chosen as both most liked and least liked. Although preferences can be neither right nor wrong, an individual is likely to consider it an error to be inconsistent in that way, even though a signal-detection model predicts such inconsistencies to occur. Inconsistent choices were made by 34 (35\%) of the 96 subjects, and consistent choices were made by $65 \%$. The pattern of performance on the other tasks did not differ significantly between the consistent and the inconsistent subjects, arguing that they did not constitute distinct groups differing in reliability of judgment but only exhibited different outcomes from the same underlying processes. Because the subjects who had triangles containing two tap water samples in $C$ had only 1 tap water sample in $D$, and vice versa, the chance expectation of consistent choices is $44.4 \%$. The observed excess of consistency over chance is highly significant $\left(\chi^{2}=18.26, p<.001\right)$.

Columns $\mathrm{A}$ and $\mathrm{C}$ with triangle type 1T-2D correspond to the conditions studied by MacRae and Geelhoed (1992), and the results here are similar, with only 20 subjects (42\%) correctly identifying tap water as the odd sample and 32 subjects $(67 \%)$ giving it as most preferred $\left(\chi^{2}=\right.$ $6.04, p<.02$, for the difference). The converse situation occurs in columns $\mathrm{A}$ and $\mathrm{D}$ with triangle type $2 \mathrm{~T}$ $1 \mathrm{D}$, though the effect is weaker, with $42 \%$ correctly identifying distilled water as the odd sample and $58 \%$ giving it as the least preferred $\left(\chi^{2}=2.67\right)$.

From the whole of column A, we see that 40 subjects $(42 \%)$ correctly selected the odd sample, compared with a chance expectation of $33 \%\left(\chi^{2}=3.00\right)$. Column B represents the choices when asked for what was either liked most or liked least. In that task, where two of the samples are of the same material, the odd sample should be

Table 1

Frequencies of Choice of Tap and Distilled Water in Four Tasks, A-D, With Triangles Containing Two Samples of Tap Water (2T-1D) or One Sample of Tap Water (1T-2D)

\begin{tabular}{|c|c|c|c|c|c|}
\hline Triangle Type & Choice & $\frac{A}{\text { Oddity }}$ & $\frac{\mathrm{B}}{\begin{array}{c}\text { Most or } \\
\text { Least }\end{array}}$ & $\frac{C}{\text { Most }}$ & $\frac{\mathrm{D}}{\text { Least }}$ \\
\hline $2 T-1 D$ & & & Exosit & 35 & 20 \\
\hline $21-10$ & distilled & $\begin{array}{l}28 \\
20\end{array}$ & $\begin{array}{l}23 \\
23\end{array}$ & $\begin{array}{l}53 \\
13\end{array}$ & 28 \\
\hline $1 T-2 D$ & $\begin{array}{l}\text { tap } \\
\text { distilled }\end{array}$ & $\begin{array}{l}20 \\
28\end{array}$ & $\begin{array}{l}21 \\
27\end{array}$ & $\begin{array}{l}32 \\
16\end{array}$ & $\begin{array}{l}13 \\
35\end{array}$ \\
\hline
\end{tabular}


liked either most or least, so it is reasonable to regard selection of a member of the pair as an error in Task B as well as in Task A. On that view, $44(45 \%)$ of the choices were correct, again with a chance expectation of $33 \%\left(\chi^{2}=6.75\right)$. Although correct choices were more numerous than in Task $A$, the difference between the proportions correct was far from being significant $\left(\chi^{2}=\right.$ $0.17)$.

\section{DISCUSSION}

Task A was explicitly expressed in terms of oddity, whereas Task B was expressed in terms of preference, but they were performed with about equal consistency. It thus appears that the difference between oddity and preference tasks is not crucial. Task B left the direction of preference unspecified, whereas Tasks $C$ and $D$, which also involved preference, specified the direction and were performed with greater consistency. Thus, the difference between the two conditions of our earlier experiment should not be attributed to the fact that one involved preference while the other involved detection of oddity. Rather, it relates to the fact that in the oddity task the difference to be detected is not specified.

Frijters $(1979 a, 1979 b)$ proposed a model for the triangular test-following David and Trivedi (1962) and Ura (1960)-which predicts a greater success rate for the 3AFC than for the triangular test if performance is above chance. In the 3-AFC, the optimal decision rule is to select the item with greatest magnitude; however, in the triangular test, the optimal rule is to select the item not participating in the smallest sensory distance between pairs of magnitudes. The distinction is between what Macmillan and Creelman (1991) call, respectively, an "independent observations" decision rule and a "differencing" decision rule. The differencing rule results in a smaller proportion of correct responses for any $d^{\prime}$ above zero.

Although simpler expressions are possible, the corresponding expressions for the expected proportions of odd selections with one odd and two identical samples are shown in forms close to those in Frijters, Kooistra, and Vereijken (1980). For the differencing rule,

$$
\begin{aligned}
p(\text { odd })= & 2 \int_{0}^{\infty}\left[\Phi\left(-u \sqrt{3}+d^{\prime} \sqrt{2 / 3}\right)\right. \\
& \left.+\Phi\left(-u \sqrt{3}-d^{\prime} \sqrt{2 / 3}\right)\right] \phi(u) d u,
\end{aligned}
$$

and for the independent observations rule,

$$
p(\text { odd })=\int_{0}^{\infty}\left[\Phi^{2}\left(u+d^{\prime}\right)+\Phi^{2}\left(-u+d^{\prime}\right)\right] \phi(u) d u,
$$

where $\phi$ is the ordinate of the unit normal curve at $u$, and $\Phi$ is its area below $u$.

These models, respectively, can be applied to Tasks A and $B$ (analogous to the triangle test since the attribute distinguishing the odd sample was unspecified) and Tasks $C$ and D (analogous to 3-AFC since the distinguishing attribute was specified). To locate the odd item without a
Table 2

Discrimination Index, $d^{\prime}$, for Each Task and Triangle Type,

\begin{tabular}{|c|c|c|c|c|}
\hline \multirow[b]{3}{*}{ Triangle Type } & \multirow[b]{2}{*}{ A } & \multirow{2}{*}{$\frac{B}{\text { Most or }}$} & \multirow[b]{2}{*}{ C } & \multirow[b]{2}{*}{ D } \\
\hline & & & & \\
\hline & Oddity & Least & Most & Least \\
\hline $2 T-1 D$ & 0.99 & 1.36 & 0.23 & 0.83 \\
\hline IT-2D & 0.99 & 1.11 & 1.11 & 0.23 \\
\hline
\end{tabular}
Assuming That Tasks $A$ and $B$ Use the Triangle Decision Rule and Tasks C and D Use the 3-AFC Rule

specific characteristic to look for, as in Tasks A and B, the subject locates the smallest of the three sensory distances and chooses as the odd item the sample not involved. When a characteristic is specified, as in Tasks $\mathrm{C}$ and D, the subject chooses the sample that has most.

The models predict the greater consistency we found for preference judgments, provided that preferences are mainly in one direction. The values of $d^{\prime}$ shown in Table 2 are from the tables in Hacker and Ratcliff (1979) for 3-AFC and the normal-model tables in Frijters et al. (1980) for the triangular test. The calculation for the oddity model is obvious with Tasks A and B. For example, the proportion of selections of the odd item, $p$ (odd), is $20 / 48$ or 0.417 for both triangle types in Task A. It is also obvious for Task $C$ when the tap water is the odd one and for Task $\mathrm{D}$ when distilled is the odd one, with $p$ (odd) being $32 / 48$ or 0.667 and $28 / 48$ or 0.583 , respectively. It is less obvious when the most liked sample has to be selected from two of the preferred type, or the least liked has to be selected from two of the less liked type. But in these cases, too, we can consider the probability of picking the odd sample, because picking one of the two "correct" samples amounts to avoiding the odd one. For Task C and 2T-1D, and also for Task D and 1T-2D, $p$ (odd) is $13 / 48$ or 0.271 . The tabled $d^{\prime}$ is -0.23 for picking the odd sample, so $d^{\prime}$ for avoiding it is 0.23 . That is much lower than any of the other $d$ 's and may indicate that the subjects found that task troublesome and unnatural. However, another possibility is discussed later.

The mean of the eight resulting $d^{\prime} s$ is 0.856 . Taking that as an estimate of the underlying discriminability of the two waters and translating back again as shown in Table 3 yields a set of expected frequencies predicted by the model if no effect other than the different decision rules is at work. The fit of the observations to the model is good for six of the conditions but poor for the conditions that also yielded the lowest estimates of $d^{\prime}-$ that is, those where the most liked had to be chosen from two tap water samples or the least liked had to be chosen from two distilled. Each of these conditions contributes 7.38 to the overall $\chi^{2}$ of 17.76. With 7 degrees of freedom, $p<.05$. The best fit is obtained with $d^{\prime}=0.67$, but the $\chi^{2}$ of 14.57 is still significant at $p<.05$, indicating a poor overall fit.

We conclude that the models as stated above offer a good account of the disparity between our original preference and oddity tasks but do not completely explain 
Table 3

Expected Response Frequencies if $d^{\prime}=\mathbf{0 . 8 5 6}$ (the Mean of the Entries in Table 2, and With the Same Assumptions)

\begin{tabular}{|c|c|c|c|c|c|}
\hline \multirow[b]{3}{*}{ Triangle Type } & \multirow[b]{3}{*}{ Choice } & \multirow[b]{2}{*}{$\mathrm{A}$} & $\mathrm{B}$ & \multirow[b]{2}{*}{$\mathrm{C}$} & \multirow[b]{2}{*}{ D } \\
\hline & & & $\overline{\text { Most or }}$ & & \\
\hline & & Oddity & Least & Most & Least \\
\hline \multirow[t]{2}{*}{$2 \mathrm{~T}-1 \mathrm{D}$} & & 28.96 & 28.96 & 41.46 & 19.62 \\
\hline & distilled & 19.04 & 19.04 & 6.54 & 28.38 \\
\hline \multirow[t]{2}{*}{$1 T-2 D$} & tap & 19.04 & 19.04 & 28.38 & 6.54 \\
\hline & distilled & 28.96 & 28.96 & 19.62 & 41.46 \\
\hline
\end{tabular}

the results when subjects have to choose a most preferred sample from a set containing two identical samples that are preferred to the third, or to choose their least preferred when the identical samples are liked less than the third.

The results from Tasks C and D show that preference is predominantly for tap water, with only about a third of the preferences being for distilled water. If we focus only on the individuals whose choices in C and D were consistent-that is, who chose tap as most preferred and also chose distilled as least preferred, or vice-versa-the ratio is about 3 to 1 in favor of tap. However, it is not a necessary consequence of these results that some subjects have a consistent tendency to prefer one water while some prefer the other. If our models are correct, both types of response are expected to occur even if all subjects have the same direction of weak preference. As a result, it is not legitimate to identify even those who give consistent responses on Tasks $C$ and $D$ as necessarily having a corresponding tendency to prefer that particular type of water. (Even less would it be legitimate to analyze results from Tasks C and D for groups selected on the basis of their performance on these tasks.) Thus, although it would be desirable to focus on individuals and characterize them for the purpose of further analysis by the direction of their preferences, that is not an option with the present data.

However, we can examine the model that all subjects can be characterized by a single magnitude of $d^{\prime}$ (representing their equal sensitivity to the difference between tap and distilled water) but a minority prefer distilled water to tap water. For the minority group, $d^{\prime}$ effectively

Table 4

Expected Response Frequencies if $d^{\prime}=1.3$ and 25\% of the Subjects Prefer Distilled Water

\begin{tabular}{|c|c|c|c|c|c|}
\hline \multirow[b]{3}{*}{ Triangle Type } & \multirow[b]{3}{*}{ Choice } & \multicolumn{2}{|r|}{ B } & \multirow[b]{2}{*}{$\mathrm{C}$} & \multirow[b]{2}{*}{$\mathrm{D}$} \\
\hline & & A & Most or & & \\
\hline & & Oddity & Least & Most & Least \\
\hline \multirow[t]{2}{*}{$2 T-1 D$} & & 25.49 & 25.49 & 36.73 & 21.32 \\
\hline & distilled & 22.51 & 22.51 & 11.27 & 26.68 \\
\hline \multirow[t]{2}{*}{$1 \mathrm{~T}-2 \mathrm{D}$} & tap & 22.51 & 22.51 & 26.68 & 11.27 \\
\hline & distilled & 25.49 & 25.49 & 21.32 & 36.73 \\
\hline
\end{tabular}

has the opposite sign since, for example, selection of a tap water sample under instructions to select the most preferred is an error, whereas for the majority it is a success. This has no implication for the triangular test because the reason for selecting an odd sample is immaterial; however, it affects the 3-AFC predictions.

Table 4 shows the predictions of a model where $25 \%$ of the subjects prefer distilled water to tap water and all subjects have $d^{\prime}=1.3$ for the difference between the water types. For the present data, these values are close to the minimum $\chi^{2}$ values of their parameters, obtained by manual search using a numerical procedure implemented in Mathcad 4.0 for Windows. ${ }^{1}$

For $d^{\prime}=1.3, p(c)$ under the differencing rule is 0.531 , so the expectation is 25.49 out of 48 successful selections of the odd sample in each version of Tasks A and B. In Task $C$ with $2 \mathrm{~T}-1 \mathrm{D}$, we consider the number expected to select distilled water using the independent observations rule. For the majority ( 36 who prefer tap water out of 48), $d^{\prime}$ for selecting distilled water as most preferred is -1.3 , with a consequent $p$ (odd) of .0743 . For the minority group (12 who prefer distilled water out of 48 ), $d^{\prime}$ for selecting distilled water is 1.3 , with a consequent $p$ (odd) of .7163. The expected number selecting distilled water in that condition is therefore $36 \times .0743+12 \times$ $.7163=11.27$, and the number selecting tap water is 48 - 11.27. Analogous calculations yield the other entries in Table 4.

The expected frequencies in Table 4 give an excellent fit to the data, with departure from the model giving $\chi^{2}$ $=4.50$ with 6 degrees of freedom $(p>.5)$. Although the model fits well, this demonstrates only that it can account for the results, not that it is the only possible account. For example, it seems unlikely that individuals are truly identical in discriminating power.

Our main conclusion, however, is that it is not necessary to invoke any advantage for hedonic judgments to explain our earlier results. These, and the new results here, are just particular instances of the advantage in statistical power that Ennis (1990) shows forced-choice methods to have over triangular tests. More consistent judgments are a consequence not of greater sensitivity to hedonic differences but to the statistical properties of the decision rules followed in different tasks.

\section{REFERENCES}

David, H. A., \& Trivedi, M. C. (1962). Pair, triangle and duo-trio tests (Tech. Rep. No. 55). Blacksburg, VA: Virginia Polytechnic Institute, Department of Statistics.

ENNis, D. M. (1990). Relative power of difference testing methods in sensory evaluation. Food Technology, 44, 114-117.

Fruters, J. E. R. (1979a). The paradox of discriminatory nondiscriminators resolved. Chemical Senses \& Flavour, 4, 355-358.

FrIJTERs, J. E. R. (1979b). Variations of the triangular method and the relationship of its unidimensional probabilistic models to threealternative forced-choice signal detection theory models. British Journal of Mathematical \& Statistical Psychology, 32, 229-241. 
Frijters, J. E. R., Kooistra, A. \& Vereijken, P. F. G. (1980). Tables of $d^{\prime}$ for the triangular method and the 3-AFC signal detection procedure. Perception \& Psychophysics, 27, 176-178.

HACKER, M. J., \& RATCLIFF, R. (1979). A revised table of $d^{\prime}$ for Malternative forced choice. Perception \& Psychophysics, 26, 168-170.

Macmillan, N. A., \& Creelman, C. D. (1991). Detection theory: $A$ user's guide. Cambridge: Cambridge University Press.

MacRae, A. W., \& Geelhoed, E. N. (1992). Preference can be more powerful than detection of oddity as a test of discriminability. Perception \& Psychophysics, 51, 179-181.

URA, S. (1960). Pair, triangle and duo-trio tests. Reports of Statistical Application Research, Union of Japanese Scientists \& Engineers, 7, 107-119.

\section{NOTE}

1. A copy of the routine used, which requires Mathcad 4.0 for Windows, can be obtained on request with a formatted 5.25- or 3.5-in. PCcompatible disk to A. W. MacRae, School of Psychology, University of Birmingham, Edgbaston, Birmingham, B15 2TT, U.K.

(Manuscript received November 12, 1991; revision accepted for publication August 17, 1993.) 